\title{
Light Cone Expansion and Four Point Function`
}

\author{
J. Kühn and E. Seiler \\ Max-Planck-Institut für Physik und Astrophysik, Munich, Federal Republic of Germany
}

Received April 12, 1973

\begin{abstract}
The behaviour of products of local fields for lightlike distances is investigated. If a light cone expansion of $A(x) A(y)$ exists, then already the four point function carries the singularity arising in the expansion for $(x-y)^{2} \rightarrow 0$. For a special class of field theories, discussed by S. Schlieder and E. Seiler, it is shown that the light cone expansion is possible.
\end{abstract}

\section{Introduction}

The close connection between short-distance expansion (SDE) and light cone expansion (LCE) has been pointed out by several authors [1-3]. This suggests the idea to derive the LCE from the SDE just by using Lorentz invariance, but so far only heuristic arguments have been given. Using similar methods as Schlieder and Seiler [4] in a recent paper on the SDE we prove

1. If a LCE of $A(x) A(y)$ exists, then the leading light cone singularity occurs already in the four point function.

$\star$ Notation. $\mathscr{S}\left(\mathbb{R}^{n}\right)$ the Schwartz space of strongly decreasing testfunctions over $\mathbb{R}^{n}$ $A=$ scalar field operator, which fulfils the Wightman axioms [we freely write $A(x), x \in \mathbb{R}^{4}$; and $\left.A(g), g \in \mathscr{S}\left(\mathbb{R}^{4}\right)\right]$.

$\mathscr{H}=$ Hilbert space.

$$
\begin{gathered}
\zeta, z \in \mathbb{C}^{4} ; \quad x, y, \xi, \eta \in \mathbb{R}^{4} ; \\
\zeta:=\left(\zeta_{1}, \ldots, \zeta_{n}\right) ; \quad \xi:=\left(\zeta_{1}, \ldots \zeta_{J-1}, \zeta_{J+1}, \ldots \zeta_{n}\right) ; \\
V_{ \pm}:=\left\{\eta \mid \eta^{2}>0, \eta_{0} \gtrless 0\right\} ; \\
\tau_{ \pm}:=\left\{\zeta \mid \zeta=\xi+i \eta, \eta \in V_{ \pm}\right\} ;
\end{gathered}
$$

$\Omega=$ vacuum state.

$\mathcal{D}=\left\{\phi \in \mathscr{H} \mid \phi=\left(\sum_{k=1}^{n} \int A\left(x_{1}\right) \ldots A\left(x_{k}\right) f_{k}(\underline{x}) d \underline{x}+f_{0}\right) \Omega ; f_{k} \in \mathscr{S}\left(\mathbb{R}^{4 k}\right)\right\}$.

$\mathfrak{I} \subset \mathfrak{D}$ is the linear hull of the vectors

$$
\Omega ; A\left(z_{1}^{1}\right) \Omega ; \ldots ; A\left(z_{1}^{n}\right) A\left(z_{2}^{n}\right) \ldots . A\left(z_{n}^{n}\right) \Omega ;\left(z_{1}^{k}, z_{j}^{k}-z_{j-1}^{k} \in \tau_{+}\right) .
$$

(With respect to the definition of operators with complex argument cf. [6]!)

$$
\mathscr{W}_{n}\left(x_{1}, \ldots, x_{n}\right):=W_{n}\left(x_{2}-x_{1}, \ldots, x_{n}-x_{n-1}\right):=\left(\Omega, A\left(x_{1}\right) \ldots . A\left(x_{n}\right) \Omega\right) .
$$

By $\delta_{\varrho}\left(x^{2}\right)$ we denote a sequence of functions $(\in \mathscr{P})$ which converges to $\delta\left(x^{2}\right)$ as $\varrho \rightarrow 0$. 
2. For a special class of Wightman fields, discussed by Schlieder and Seiler, the LCE exists.

II.

Lemma. We assume the existence of a LCE in the following form: There is a function $r(\xi)$ such that $C(f, g):=\int r(\xi) A(x) A(x+\xi) f(x)$ $\cdot g(\xi) d x d \xi$ exists as an operator valued distribution on $\mathfrak{D}$ for $f, g \in \mathscr{S}$, $\operatorname{supp}(g) \subset \mathfrak{U}_{R}(L C)$ (where $\mathfrak{U}_{R}(L C):=\left\{x\left|x \in \mathbb{R}^{4} ;\right| x^{2} \mid<R^{2}\right\}$ ) and which allows the "restriction of $C$ to the light cone," that is

$$
(\psi, C(f, g) \varphi)_{L C}:=\lim _{\varrho \rightarrow 0}\left(\psi, C\left(f, g \cdot \delta_{\varrho}\right) \varphi\right) \quad\left(g \cdot \delta_{\varrho}(\xi):=g(\xi) \delta_{\varrho}\left(\xi^{2}\right)\right)
$$

exists for all $\psi, \varphi \in \mathfrak{D}$.

Then from

$$
\begin{aligned}
\lim _{\varrho \rightarrow 0} \int \mathscr{W}_{4}\left(x^{\prime}+\xi^{\prime}, x^{\prime}, x, x+\xi\right) \bar{r}\left(\xi^{\prime}\right) r(\xi) \bar{f}\left(x^{\prime}\right) \bar{g}\left(\xi^{\prime}\right) f(x) g(\xi) \delta_{\varrho}\left(\xi^{\prime 2}\right) \\
\cdot \delta_{\varrho}\left(\xi^{2}\right) d x d x^{\prime} d \xi d \xi^{\prime} \\
=\lim _{\varrho \rightarrow 0}\left(C\left(f, g \delta_{\varrho}\right) \Omega, C\left(f, g \cdot \delta_{\varrho}\right) \Omega\right)=0
\end{aligned}
$$

follows

$$
(\psi, C(f, g) \varphi)_{L C}=0, \quad \varphi, \psi \in \mathfrak{D}^{1} .
$$

Proof. From (2) follows for $\psi \in \mathfrak{D}$

$$
\begin{aligned}
\left|(\psi, C(f, g) \Omega)_{L C}\right| & =\lim _{\varrho \rightarrow 0}\left|\left(\psi, C\left(f, g \cdot \delta_{\varrho}\right) \Omega\right)\right| \\
& \leqq \lim _{\varrho \rightarrow 0}\|\psi\| \cdot\left\|C\left(f, g \cdot \delta_{\varrho}\right) \Omega\right\|=0 .
\end{aligned}
$$

Obviously $C(f, g)_{L C}$ is a bilocal bilinear form, that is it commutes with $A(h)$ if $\operatorname{supp}(h)$ is spacelike to $\operatorname{supp}(f)$ and $\operatorname{supp}(f)+\operatorname{supp}(g)$. Since $\Omega$ is a separating vector also for bilinear forms ${ }^{2}$, Eq. (3) follows.

The lemma can also be stated in a slightly different form: Under the assumptions mentioned $\mathscr{W}_{4}\left(x_{1}, \ldots, x_{4}\right)$ is as singular as $\left(r\left(x_{1}-x_{2}\right)\right)^{-1}$ $\cdot\left(r\left(x_{3}-x_{4}\right)\right)^{-1}$ if $\left(x_{1}-x_{2}\right)^{2}$ and $\left(x_{3}-x_{4}\right)^{2}$ go to 0 simultaneously.

If instead of Eq. (2) we assume the existence of

$$
w-\lim _{\varrho \rightarrow 0} C\left(f, g \cdot \delta_{\varrho}\right) \psi=: C(f, g)_{L C} \psi ; \psi \in \mathfrak{D}
$$

it follows that $\mathscr{W}_{4}\left(x_{1}, \ldots, x_{4}\right)$ for $\left(x_{3}-x_{4}\right)^{2} \rightarrow 0$ is as singular as $\left(r\left(x_{3}-x_{4}\right)\right)^{-1}$. The proof is completely analogous to the proof of the corresponding statement for $\left(x_{3}-x_{4}\right) \rightarrow 0$ (see Ref. [4], Section 2, Lemma 1).

${ }^{1}$ A similar statement is true if we consider $C_{1}=r_{1}(\xi)\left(A(x) A(x+\xi)-W_{2}(\xi)\right)$ and $\mathscr{W}_{4}\left(x^{\prime}+\xi^{\prime}, x^{\prime}, x, x+\xi\right)-W_{2}\left(\xi^{\prime}\right) W_{2}(\xi)$ instead of $C$ and $W_{4}$.

2 The proof is similar as in Ref. [4], Appendix 1. 
III.

We intend now to prove the LCE of $A(x) A(y)$ under the conditions on the four-point function which are formulated in Ref. [4]. We are going to use the following theorem which has been proved in Ref. [4]:

We assume

(A) A function $r(\zeta)$ exists with the following properties:

a) $r$ is holomorphic in $\tau_{+}$;

b) $r(\zeta)=\overline{r(-\bar{\zeta})}$;

c) $r$ is invariant under the (homogeneous) Lorentz group;

d) $W_{4}\left(\zeta_{1}, \zeta_{2}, \zeta_{3}\right) r\left(\zeta_{3}\right)$ has (a locally unique) analytic continuation to the points $\left(\zeta_{1}, \zeta_{2}\right) \in \tau_{+}^{(2)}, \zeta_{3} \in \mathfrak{U}_{R}(0)$ where $\mathfrak{U}_{R}(0):=\{\xi|| \xi \mid<R\}$ is independent of $\zeta_{1}, \zeta_{2}$;

e) there exists an integer $N>0$ such that for each compact subset $K \subset V_{+}, \xi \in \mathfrak{U}_{R}(0), \eta / \sqrt{\eta^{2}} \in K, r$ fulfils the following inequality

$$
|r(\xi+i \eta)| \leqq\left(\eta^{2}\right)^{-N} C_{K} \quad\left(0<\eta^{2} \leqq 1\right)
$$

$\left(C_{K}\right.$ is a constant depending on $\left.K\right)$.

Then $F_{j}(\xi):=W_{n}(\xi) \cdot r\left(\zeta_{j}\right)$ has a unique analytic continuation to the points $\zeta_{j}=0, \zeta_{i} \in \tau_{+}^{(n-2)}$. Furthermore $F_{j}$ is holomorphic for $\xi_{j} \in \mathfrak{U}_{R^{\prime}}(0)$, $\xi \in \tau_{+}^{(n-2)}$ where $R^{\prime}>0$ is independent of $\xi^{3}$.

Remark. In general it is impossible to find a complex neighborhood $\mathfrak{B}$ of 0 independent of $\xi$, such that $F_{j}$ is holomorphic in $\tau_{+}^{(n-2)} \times \mathfrak{B}$, as can be seen from the free mass zero field:

$$
\begin{aligned}
& r\left(\zeta_{1}\right) W_{4}(\xi)=\zeta_{1}^{2} W_{4}(\zeta) \\
& =\frac{1}{\zeta_{3}^{2}}+\frac{\zeta_{1}^{2}}{\zeta_{2}^{2}\left(\zeta_{1}+\zeta_{2}+\zeta_{3}\right)^{2}}+\frac{\zeta_{1}^{2}}{\left(\zeta_{1}+\zeta_{2}\right)^{2}\left(\zeta_{2}+\zeta_{3}\right)^{2}}
\end{aligned}
$$

is holomorphic for $\zeta_{1} \in \mathbb{R}^{4} ; \zeta_{2}, \zeta_{3} \in \tau_{+}$; but for every $\zeta_{1} \in \tau_{-}$there are $\zeta_{2}, \zeta_{3} \in \tau_{+}$such that $r W_{4}$ is singular.

Now we prove the following

Theorem. Under the assumptions $(A)$

$$
C(x, \xi):=r(\xi) A(x) A(x+\xi)
$$

defines a bilocal operator with the following properties:

a) $C(f, g)=\int C(x, \xi) f(x) g(\xi) d x d \xi$ is for $f, g \in \mathscr{S}, \operatorname{supp}(g) \subset \mathfrak{U}_{R}(L C)$ defined on $\mathfrak{D}$ and $C(f, g) \mathfrak{D}$ lies in the domain of definition of $\bar{A}$, the closure of $A^{4}$.

b) $[C(f, g), A(h)]=0$ if $\operatorname{supp}(h)$ is spacelike to $\operatorname{supp}(f) \cup(\operatorname{supp}(f)$ $+\operatorname{supp}(g))$. That means $C$ is a "bilocal" operator.

${ }^{3}$ Ref. 4, Section 3, Lemma 3.

${ }_{4} \bar{A}$ exists, because the adjoint of $A$ is densely defined. 
c) For fixed $\varphi, \psi \in \mathfrak{J}$ the matrix element $(\psi, C(x, \xi) \varphi)$ is holomorphic for $x \in \mathbb{R}^{4}, \xi \in \mathfrak{U}_{R^{\prime}}(L C)$, where $R^{\prime}>0$ is suitably chosen.

Remark. The last statement is not true for $\xi$ in a neighborhood of the complex light cone as can be seen again from the free mass zero field: We choose in the example considered above

$$
\zeta_{1}=i(-1,1,0,0) ; \quad \zeta_{2}=\zeta_{3}=i(1,0,0,0) \in \tau_{+} .
$$

Proof of the Theorem. a) We define $C(f, g)$ on $\psi$

$$
\psi:=\int A\left(x_{1}\right) \cdots \cdot A\left(x_{n}\right) \Omega h(\underline{x}) d \underline{x} ; \quad\left(h \in \mathscr{S}\left(\mathbb{R}^{4 n}\right)\right)
$$

as follows:

$$
\begin{aligned}
\psi_{\eta}:= & \int A\left(x_{1}+i \eta_{1}\right) \ldots A\left(x_{n}+i \eta_{n}\right) \Omega h(\underline{x}) d \underline{x} \\
& \eta_{1} \in V_{+}, \eta_{j+1}-\eta_{j} \in V_{+} ; \quad(j=1, \ldots n-1), \\
\varphi_{y, \eta, \eta}:= & \int A(x+i y) A(x+\xi+i y+i \eta) \cdot r(\xi+i \eta) \psi_{\eta} f(x) g(\xi) d x d \xi \\
& y, \eta, \eta_{1}-\eta-y \in V_{+} ; \eta_{j+1}-\eta_{j} \in V_{+} ; \quad(j=1, \ldots, n-1) .
\end{aligned}
$$

Obviously $\psi_{\eta}, \varphi_{y, \eta, \eta} \in \mathfrak{D}$.

For $y, \eta, \eta \rightarrow 0 \varphi_{y, \eta, \eta}$ converges strongly:

$$
\begin{array}{r}
\left\|\varphi_{y^{\prime}, \eta^{\prime}, \eta^{\prime}}-\varphi_{y, \eta, \eta}\right\|^{2}=\left(\varphi_{y, \eta ; \eta}, \varphi_{y, \eta, \eta}\right)+\left(\varphi_{y^{\prime}, \eta^{\prime}, \eta^{\prime}}, \varphi_{y^{\prime}, \eta^{\prime}, \eta^{\prime}}\right) \\
-\left(\varphi_{y^{\prime}, \eta^{\prime}, \eta^{\prime}}, \varphi_{y, \eta, \eta}\right)-\left(\varphi_{y, \eta, \eta}, \varphi_{y^{\prime}, \eta^{\prime}, \eta^{\prime}}\right) \rightarrow 0 \\
\left(y, y^{\prime}, \eta, \eta^{\prime}, \eta, \eta^{\prime} \rightarrow 0\right)
\end{array}
$$

since each term converges to the same limit, which exists because of assumption e) according to Appendix 2 of Ref. [4].

We then define

$$
C(f, g) \psi:=\underset{y, \eta, \eta \rightarrow 0}{s-\lim _{y, \eta, \eta}} \varphi
$$

Now $\varphi_{y, \eta, \eta} \in \mathfrak{D}$ and $A\left(f_{1}\right) \varphi_{y, \eta, \eta} f_{1} \in \mathscr{S}$ converge strongly for $y, \eta, \eta \rightarrow \underline{0}$ (this can be seen in the same way as the convergence of $\varphi_{y, \eta, \eta}$ ). Hence $\bar{A}$ is defined on $C \mathfrak{D}$.

b) The "bilocality" of $C$ is a trivial consequence of the locality of $A$ and the fact that the Wightman distributions can be multiplied by $r$.

c) The properties a) and b) are true without assumption e). This assumption, however, is essential if one wants to restrict $\xi$ to the light cone. In the theorem of Ref. [4] it is proved that $F_{j}$ is holomorphic for $\xi_{j} \in \mathfrak{U}_{R^{\prime}}(0), \xi \in \tau_{+}^{(n-2)}$. If we now choose a $\xi_{j}^{\prime} \in \mathfrak{U}_{R^{\prime}}(L C)$ we can find a real Lorentz transformation $\Lambda$ such that $\Lambda \xi_{j}^{\prime} \in \mathfrak{U}_{R^{\prime}}(0)$, since the Lorentz group acts transitively on the hyperboloids $\xi_{j}^{2}=$ const. But $F_{j}$ is Lorentz invariant, hence it is holomorphic in $\mathfrak{U}_{R^{\prime}}(L C) \times \tau_{+}^{(n-2)}$. 
If now $\varphi, \psi \in \mathfrak{I},(\psi, C(x, \xi) \varphi)$ is a finite linear combination of Wightman functions, multiplied by $r(\xi)$. Therefore we can easily construct a real neighborhood $\mathfrak{U}_{R^{\prime}}(L C)$ such that $(\psi, C(x, \xi) \varphi)$ is holomorphic in $\mathbb{R}^{4} \times \mathfrak{U}_{R^{\prime}}(L C)$.

Acknowledgements. We thank K. Baumann and S. Schlieder for useful discussions.

\title{
References
}

1. Brandt, R., Preparata, G.: Nucl. Physics B 27, 541 (1971)

2. Frishman, Y.: Annals of Physics 66, 373 (1971)

3. Zimmermann, W.: Operatorproduktentwicklungen in der Quantenfeldtheorie in: Quanten und Felder, ed. H. P. Dürr, Braunschweig: Friedr. Vieweg \& Sohn 1971

4. Schlieder, S., Seiler, E.: Commun. math. Phys. 31, 137-159 (1973)

5. Streater, R.F., Wightman, A.S.: PCT, Spin and Statistics and All that, New York: W. A. Benjamin, 1964

6. Jost, R.: The General Theory of Quantized Fields, Providence (AMS), 1965

\author{
J. Kühn, E. Seiler \\ Max-Planck-Institut \\ für Physik und Astrophysik \\ D-8000 München 40 \\ Föhringer Ring 6 \\ Federal Republic of Germany
}

